\title{
Evaluation of a multiobjective performance index for distribution systems with distributed generation
}

DOI:

10.1049/cp:20051233

Link to publication record in Manchester Research Explorer

\section{Citation for published version (APA):}

Ochoa, L. F., Padilha-Feltrin, A., \& Harrison, G. P. (2005). Evaluation of a multiobjective performance index for distribution systems with distributed generation. In IEE Conference Publication/IEE Conf Publ (Vol. 4, pp. 175-178) https://doi.org/10.1049/cp:20051233

\section{Published in:}

IEE Conference Publication|IEE Conf Publ

\section{Citing this paper}

Please note that where the full-text provided on Manchester Research Explorer is the Author Accepted Manuscript or Proof version this may differ from the final Published version. If citing, it is advised that you check and use the publisher's definitive version.

\section{General rights}

Copyright and moral rights for the publications made accessible in the Research Explorer are retained by the authors and/or other copyright owners and it is a condition of accessing publications that users recognise and abide by the legal requirements associated with these rights.

\section{Takedown policy}

If you believe that this document breaches copyright please refer to the University of Manchester's Takedown Procedures [http://man.ac.uk/04Y6Bo] or contact uml.scholarlycommunications@manchester.ac.uk providing relevant details, so we can investigate your claim.

\section{OPEN ACCESS}




\title{
EVALUATION OF A MULTIOBJECTIVE PERFORMANCE INDEX FOR DISTRIBUTION SYSTEMS WITH DISTRIBUTED GENERATION
}

\author{
Luis F. OCHOA* ${ }^{*}$, Antonio PADILHA-FELTRIN*, Gareth P. HARRISON $\dagger$ \\ * UNESP - Universidade Estadual Paulista, Campus de Engenharia de Ilha Solteira - Brazil \\ $\dagger$ University of Edinburgh, School of Engineering and Electronics - United Kingdom \\ luis_ochoa@ieee.org, padilha@dee.feis.unesp.br, gareth.harrison@ed.ac.uk
}

\begin{abstract}
SUMMARY
Here a multiobjective performance index for distribution systems with distributed generation based on a steady-state analysis of the network is proposed. This index quantifies the distributed generation impact on total losses, voltage profile and short circuit currents, and will be used as objective function in an evolutionary algorithm aimed at searching the best points for connecting distributed generators. Moreover, a loss allocation technique, based on the Zbus method, is applied on the original configuration of the network to obtain a good quality initial population. An IEEE medium voltage distribution network is analysed and results are presented and discussed.
\end{abstract}

\section{DISTRIBUTED GENERATION}

Defined as electric power generation within distribution networks or on the costumer side of the meter [1], distributed generation (DG) technologies include photovoltaics, wind turbines, internal combustion engines, combustion turbines, microturbines and fuel cells, among others. Encouraged by energy policies in many countries due to environmental and economic concerns, DG is expected to play an increasingly important role in the electric power system infrastructure and market. Nevertheless, various studies have demonstrated that integration of DG in distribution networks may create technical and safety problems [2-5]. The main issues include where to locate and how to operate DG to minimise the impact on distribution management. Additionally, it is necessary to investigate whether DG capability and placement could be used to enhance distribution network planning and operation [6-10]. Consequently, it is critical to assess technical impacts of DG in power systems, in order to apply generators in a manner that avoids causing degradation of power quality and reliability.

\section{PROPOSED METHOD}

In this paper, technical impacts from an energy quality and reliability perspective will be assessed in a medium voltage distribution network. Here, a multiobjective performance index for distribution system with distributed generation based on a steady-state analysis of the network is proposed. This index will quantify the distributed generation impact on total losses, voltage profile and short circuit currents. Depending on the network size and on the number of generators to be connected to the network, the problem presented is of combinatorial nature. In this way an optimisation technique is required [7-8]. Here, an evolutionary algorithm (EA) for searching the best points for connecting distributed generators is used. This algorithm will possess as the objective function the proposed multiobjective index. Moreover, a loss allocation technique, based on the Zbus method, is applied in the original configuration of the distribution system in order to obtain a good quality initial population.

Determining the location for distributed generators presents, in practice, some limitations for distribution engineers. Consequently, the existence of an index based on technical impacts may assist in indicating where the distributed generation could be more beneficial for the distribution network, helping distribution engineers take decisions and even shape the nature of the contract that might be established between the utility and the distributed generator owner.

\section{Impact Indices and Multiobjective Index}

The following indices will be computed in order to describe the impacts on the network due to the presence of DG during maximum power generation. Maximum network demand will be used in all indices. Since distribution networks are inherently unbalanced due to its loads and its topology, the indices will consider phases $a, b, c$ and the neutral wire $(n)$.

For the $k$-th distribution network configuration considering DG: 1. Index related to real power losses, defined by:

$$
I L p^{k}=1-\frac{\operatorname{Re}\left\{\left.\sum_{m=1}^{N L} \bar{Z} a_{m} c_{m}\left|\bar{J} a_{m}^{k}\right|_{m}^{k}\right|^{2}+\left.\left.\bar{Z} b_{m}\left|\bar{J} n_{m}\right| \bar{J} n_{m}^{k}\right|^{2}\right|^{2}\right\}}{\operatorname{Re}\left\{\text { Losses }^{0}\right\}}
$$

where: $\bar{Z} a_{m}, \bar{Z} b_{m}, \bar{Z} c_{m}$ and $\bar{Z} n_{m}$ are self impedances of branch $m ; \bar{J} a_{m}^{k}, \bar{J} b_{m}^{k}, \bar{J} c_{m}^{k}$ and $\bar{J} b_{m}^{k}$ are currents through branch $m$ for the $k$-th distribution network configuration; $N L$ is the network number of lines; and, Losses ${ }^{0}$ is the total complex power losses for the distribution network without DG.

2. Index related to maximum voltage drop, computed by: 


$$
I V D^{k}=1-\max \left(\frac{\left|\bar{V} a_{0}\right|-\left|\bar{V} a_{i}^{k}\right|}{\left|\bar{V} a_{0}\right|}, \frac{\left|\bar{V} b_{0}\right|-\left|\bar{V} b_{i}^{k}\right|}{\left|\bar{V} b_{0}\right|}, \frac{\left|\bar{V} c_{0}\right|-\left|\bar{V} c_{i}^{k}\right|}{\left|\bar{V} c_{0}\right|}\right)_{i=1}^{N N-1}
$$

where: $\bar{V} a_{0}, \bar{V} b_{0}$ and $\bar{V} c_{0}$ are voltages at root node (equal in magnitude for the three phases); $\bar{V} a_{i}^{k}, \bar{V} b_{i}^{k}$ and $\bar{V} c_{i}^{k}$ are voltages at node $i$ for the $k$-th distribution network configuration; and, $N N$ is the network number of nodes.

3. Index related to three-phase short circuit maximum current variation in the network nodes, calculated by:

$$
I S C 3^{k}=1-\frac{\max \left(\frac{S C a b c_{i}^{k}}{S C a b c_{i}^{0}}\right)}{\frac{S C a b c_{*}^{k}}{S C a b c_{*}^{0}}}
$$

where: $S C a b c_{i}^{k}$ is the three-phase fault current value in node $i$ for the $k$-th distribution network configuration; $S C a b c_{i}^{0}$ is the three-phase fault current value in node $i$ for the distribution network without DG; $S C a b c_{*}^{k}$ and $S C a b c_{*}^{0}$ are the largest three-phase fault current value in the network for the $k$-th distribution network configuration and its correspondent for the distribution network without DG.

4. Index related to single-phase to ground short circuit maximum current variation in the network nodes, calculated by:

$$
I S C 1^{k}=1-\frac{\max \left(\frac{S C a_{i}^{k}}{S C a_{i}^{0}}, \frac{S C b_{i}^{k}}{S C b_{i}^{0}}, \frac{S C c_{i}^{k}}{S C c_{i}^{0}}\right)}{\frac{S C_{*}^{k}}{S C_{*}^{0}}}
$$

where: $S C a_{i}^{k}, S C b_{i}^{k}$ and $S C c_{i}^{k}$ are single-phase fault current value in node $i$ for the $k$-th distribution network configuration; $S C a_{i}^{0}, S C b_{i}^{0}$ and $S C c_{i}^{0}$ are single-phase fault current value in node $i$ for the distribution network without DG; and, $S C_{*}^{k}$ and $S C_{*}^{0}$ are the largest single-phase fault current value in the network for the $k$-th distribution network configuration and its correspondent for the distribution network without DG.

The first and second indices (ILp and IVD) express two closely related main benefits (when properly located) of DG: decrease of real power losses and improvement of voltage profile. Therefore, the closer indices ILP and IVD are to unity, the better the network performance is. Third and fourth indices (ISC3 and $I S C 1)$ are related to the protection and selectivity issues. These indices give the power engineer a notion of how the distributed generation is impacting on the protection devices that were planned for a network without such generation units. Hence, a low impact on this concern would be indicated by close to unity values for $I S C 3$ and $I S C 1$ indices.
In order to relate the indices the multiobjective index $(I M O)$ is proposed, which strategically gives a relevance (weighting) factor to each one. This can be performed since all impact indices were normalised, i.e. present non-dimensional values from zero to one.

$$
I M O^{k}=\left\{w_{1} I L p^{k}+w_{2} I V D^{k}+w_{3} I S C 3^{k}+w_{4} I S C 1^{k}\right\}
$$

where: $\sum_{i=1}^{4} w_{i}=1.0 \forall w_{i} \in[0,1]$

These relevance factors are intended to give the corresponding importance to each technical issue (impact indices) due to the presence of DG and depend on the required analysis (e.g. planning, regular operation, emergency operation). Table I shows the values for the relevance factors utilised in this work, considering a normal operation stage analysis. Nevertheless, since electric utilities present different concerns about losses, voltages, protection schemes, etc., those values may be modified to satisfy them. Thus, the multiobjective index will numerically describe the impact of DG, considering a given location and size, on a distribution network. Close to unity values for the multiobjective performance index means higher DG benefits.

TABLE 1 - Relevance Factors
\begin{tabular}{|c|c|c|c|}
\hline$I L p$ & $I V D$ & $I S C 3$ & $I S C 1$ \\
$w_{1}$ & $w_{2}$ & $w_{3}$ & $w_{4}$ \\
\hline 0.50 & 0.20 & 0.10 & 0.20 \\
\hline
\end{tabular}

\section{Evolutionary Algorithm}

Due to the combinatorial nature of the problem for searching the best points for inserting DG, here an evolutionary algorithm is used. This metaheuristic technique is efficient and suitable for the proposed problem [7-8].

Characteristics of the utilised EA: 1) coding: each configuration is described by a vector (chromosome) whose size is equal to the number of nodes. If a DG unit is inserted in a node, this element receives a number related to the capacity of the generator, otherwise it is zero. Elements of the chromosome for the substation and nodes fed by single- or two-phase branches are fixed to zero; 2) initial population: is created using both a reduced set of buses provided by the Zbus loss allocation method [11] (set of buses that influence the most into the total network losses) and randomly selected feasible buses; 3) genetic operators: selection is performed by tournament, single-point crossing-over and mutation with probabilities 0.7 and 0.005 , respectively, are used; 4) objective function: the EA is aimed at maximising the $I M O$, which is calculated for each configuration using described impact indices and presented relevance factors (Table 1); 5) unfeasible configurations: according to the number of DG units to be inserted some configurations may be unfeasible after the applying genetic operators, therefore those configurations will be penalised; 6) elitism: best configurations of each population will be stored in 
an elite set, which will be used as part of the selection process for the next generation; and, 7) stop criterion: when the elite set is not being updated after a given number of generations.

\section{APPLICATIONS}

The IEEE-34 four-wire three-phase medium voltage distribution network is analysed [12]. The total demand is 1770 $\mathrm{kW}$, and $72 \%$ of the loads are concentrated $56 \mathrm{~km}$ away from the root node (the most distant node is $59 \mathrm{~km}$ from the substation). Line-to-line base voltage is $\mathrm{V}_{\mathrm{b}}=24.9 \mathrm{kV}$. Simplifying, the autotransformer $24.9 \mathrm{kV} / 4.16 \mathrm{kV}$ in the original IEEE-34 test feeder is replaced with a line. The automatic voltage regulator is also not represented.

A three-phase four-wire power flow algorithm, based on the current summation backward-forward technique, described in [13], was adopted. Loads were modelled as constant power, and represent the maximum demand. Two DG units of $300 \mathrm{~kW}$ and $600 \mathrm{~kW}$ capacity (unity power factor, i.e. real power injection only), will be used for the multiobjective analysis in order to find the best insertion points in the above presented networks. Short circuit analysis was performed based on symmetrical components and considering system zero and positive sequences impedances at the substation HV/MV $Z$ sys $0=0.048+j 1.0071 \Omega$ and $Z$ sys $1=0.144+j 1.4022 \Omega$, respectively; and generators' zero, positive and negative impedances $\operatorname{Zgen} 0=j 1.6758 \Omega, \quad Z$ Zgen $1=j 6.2972 \Omega \quad$ and Zgen $2=j 3.7837 \Omega$, respectively.

Table 2 shows the 25 feasible buses (where a DG unit may be inserted) decreasingly ordered according to the value of loss allocation obtained with the Zbus method and considering the initial configuration (no DG) of IEEE-34 network. A set of buses with the highest loss allocation values are used to generate part of the initial population in the EA. After performing the proposed EA, it was found that the best insertion points for the analysed generators using the multiobjective index as objective function are nodes 12 and 19, for DG units of $300 \mathrm{~kW}$ and $600 \mathrm{~kW}$, respectively (see Figure 1). Table 3 presents 20 configurations with the highest values of multiobjective index $(I M O)$. It is important to note that neither node 12 nor 19 presented high values of loss allocation, however due to indices ISC 3 and ISC 1 related to short circuit levels, those buses with high loss allocation values and at the same time further away from the substation become nonoptimal solutions according to the specified relevance factors. A comparison of configuration 1 and 20 presented in Table 3 is made in Table 4. Here it can be verified that selecting nodes 10 and 22 (higher loss allocation than nodes 12 and 19) gives a better performance in terms of losses and voltage drop, nevertheless taking into account the short circuit levels the IMO is smaller.
TABLE 2 - IEEE-34 Feasible buses decreasingly ordered according to the value of loss allocation considering original network (no GD)

\begin{tabular}{|c|c|c|c|}
\hline pos. & node & pos. & node \\
\hline 1 & 22 & 14 & 26 \\
\hline 2 & 28 & 15 & 12 \\
\hline 3 & 25 & 16 & 8 \\
\hline 4 & 23 & 17 & 3 \\
\hline 5 & 27 & 18 & 16 \\
\hline 6 & 30 & 19 & 2 \\
\hline 7 & 15 & 20 & 1 \\
\hline 8 & 33 & 21 & 5 \\
\hline 9 & 31 & 22 & 6 \\
\hline 10 & 21 & 23 & 7 \\
\hline 11 & 10 & 24 & 17 \\
\hline 12 & 29 & 25 & 20 \\
\hline 13 & 19 & \multicolumn{2}{|c}{} \\
\cline { 2 - 3 }
\end{tabular}

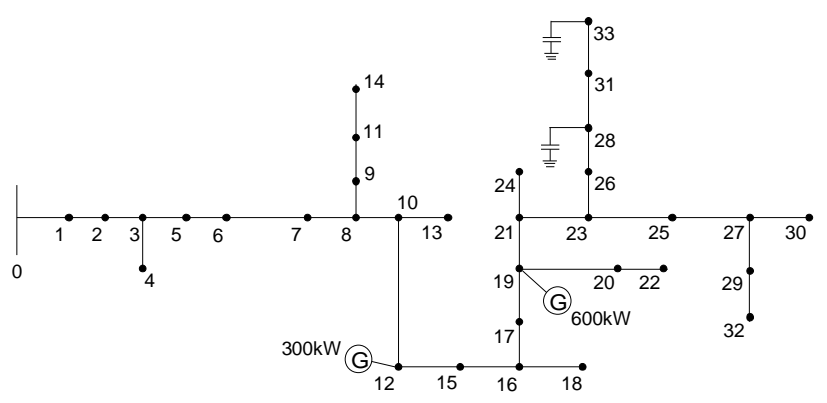

FIGURE 1 - IEEE-34 test feeder with two DG units optimally allocated using the proposed methodology.

TABLE 3 - IEEE-34 Top 20 configurations considering two different DG units and using the proposed EA.

\begin{tabular}{|c|c|c|c|c|c|c|c|}
\hline \multirow{2}{*}{ config } & \multicolumn{2}{|c|}{ nodes } & \multirow{2}{*}{$I M O$} & \multirow{2}{*}{ config } & \multicolumn{2}{|c|}{ nodes } & \multirow{2}{*}{$I M O$} \\
\hline & $300 \mathrm{~kW}$ & $600 \mathrm{~kW}$ & & & $300 \mathrm{~kW}$ & $600 \mathrm{~kW}$ & \\
\hline 1 & 12 & 19 & 0.73585 & 11 & 7 & 19 & 0.73336 \\
\hline 2 & 15 & 19 & 0.73584 & 12 & 6 & 19 & 0.73336 \\
\hline 3 & 12 & 17 & 0.73576 & 13 & 7 & 17 & 0.73328 \\
\hline 4 & 15 & 17 & 0.73575 & 14 & 6 & 17 & 0.73327 \\
\hline 5 & 10 & 19 & 0.73572 & 15 & 15 & 23 & 0.72543 \\
\hline 6 & 16 & 19 & 0.73571 & 16 & 15 & 21 & 0.72542 \\
\hline 7 & 10 & 17 & 0.73563 & 17 & 16 & 23 & 0.72539 \\
\hline 8 & 16 & 17 & 0.73562 & 18 & 16 & 21 & 0.72534 \\
\hline 9 & 8 & 19 & 0.73346 & 19 & 12 & 21 & 0.72474 \\
\hline 10 & 8 & 17 & 0.73338 & 20 & 10 & 21 & 0.7246 \\
\hline
\end{tabular}

TABLE 4 - IEEE-34 Non-normalised impact indices' comparison when inserting two DG units considering different approaches

\begin{tabular}{|c|c|c|c|c|}
\hline \multicolumn{2}{|c|}{ Impact Index } & no DG & Config. no. 1 & Config. no. 20 \\
\hline$I L p$ & $(\mathrm{~kW})$ & 397.32 & 95.40 & 92.48 \\
\hline$I V D$ & $(\%)$ & 21.21 & 10.40 & 10.19 \\
\hline$I S C 3$ & $\frac{\text { with DG }}{\text { without DG }}$ & --- & $\begin{array}{c}11.21 \\
(2451.1 \mathrm{~A} / 218.7 \mathrm{~A})\end{array}$ & $\begin{array}{c}12.13 \\
(2407.7 \mathrm{~A} / 198.5 \mathrm{~A})\end{array}$ \\
\hline$I S C I$ & $\frac{\text { with DG }}{\text { without DG }}$ & --- & $\begin{array}{c}21.77 \\
(3786.1 \mathrm{~A} / 173.9 \mathrm{~A}) \\
\end{array}$ & $\begin{array}{c}24.97 \\
(3616.5 \mathrm{~A} / 144.8 \mathrm{~A}) \\
\end{array}$ \\
\hline \multicolumn{3}{|c|}{$I M O$} & 0.73585 & 0.72460 \\
\hline \multicolumn{3}{|c|}{ Nodes with DG $(300 ; 600 \mathrm{~kW})$} & $12 ; 19$ & $10 ; 21$ \\
\hline
\end{tabular}

An optimal allocation of these generators, following the presented considerations, reduce the total network losses by $76 \%$ and the voltage drop by $51 \%$ (Table 4 ). On the other hand, the best solution increased the three and single-phase short circuit currents computed on the original configuration by 11.21 and 21.77 times, respectively (highest rates found at node 17 for both cases). These values suggest that a special attention should be paid to short circuit levels since protection schemes could be seriously affected. 
Table 3 presents different options of insertion points for the analysed two generators, regarding the described technical impacts and their respective relevance factors. However, in practice, deciding where to insert DG resources has several limitations. In fact, considering that most DG units are from cogeneration plants or renewable energies, the insertion point becomes the nearest to the resource and not necessarily to the optimal in terms of technical impacts. Therefore, presented results are intended to be used as a comparison to the actual (or pretended) insertion points of a given distributed network in which DG units will be inserted. In this way, knowing where the DG could be more beneficial will help distribution engineers take decisions and even shape the nature of the contract that might be established between the utility and the distributed generator owner.

\section{CONCLUSIONS}

Four impact indices aimed at characterising the benefits and negative impacts of DG in distribution networks were strategically related by using relevance factors in order to compute a multiobjective performance index. Values of relevance factors depend on engineers experience and utilities' different concerns about losses, voltages, protection schemes, etc., therefore the proposed methodology is flexible to any change of those values. Other technical issues, such as reactive power losses, voltage regulation, reverse power flow, etc., may be added to this approach. Also, an analysis based on both variable load demand and power generation in a diary, weekly or monthly bases could be addressed.

The multiobjective index was used as objective function of the proposed Evolutionary Algorithm aimed at providing the best configurations (set of insertion points) of a given distribution network with DG. Those results may help distribution engineers take decisions and even shape the nature of the contract that might be established between the utility and the distributed generator owner.

\section{ACKNOWLEDGEMENT}

The first author is grateful for the financial support given by FEPISA (Ilha Solteira - SP, Brazil) and the Programme Alban, the European Union Programme of High Level Scholarships for Latin America, scholarship no. E04D046001BR.

\section{REFERENCES}

[1] A. Invernizzi, B. Buchholz, M. Stubbe, N. Jenkins, B. Dowd, and M. Ceraolo, 2004, "Distribution Systems and Dispersed Generation: a New Focus for CIGRE", Electra, no. $213,17-21$.
[2] N. Jenkins, R. Allan, P. Crossley, D. Kirschen, and G. Strbac, 2000, Embedded Generation, IEE Power and Energy Series 31, London, UK, 11-18.

[3] P. P. Barker and R. W. de Mello, 2000, "Determining the impact of distributed generation on power systems: Part 1 - Radial Distribution Systems", Proceedings IEEE/PES Summer Meeting, 1645-1656.

[4] J. A. P. Lopes, 2002, "Integration of dispersed generation on distribution networks-impact studies", Proceedings IEEE/PES Winter Meeting, vol. 1, 323-328.

[5] M. T. Doyle, 2002, "Reviewing the impacts of distributed generation on distribution system protection", Proceedings IEEE/PES Summer Meeting, vol. 1, 103-105.

[6] P. Espie, G. W. Ault, G. M. Burt, and J. R. McDonald, 2003, "Multiple criteria decision making techniques applied to electricity distribution system planning", IEE Proc. - Gen., Trans. and Dist., vol. 150, no. 5, 527-535.

[7] A. Silvestri, A. Berizzi, and S. Buonanno, 1999, "Distributed generation planning using genetic algorithms", Proceedings IEEE PowerTech, 257.

[8] G. Celli and F. Pilo, 2001, "MV network planning under uncertainties on distributed generation penetration", Proceedings IEEE/PES Summer Meeting, vol. 1, 485-490.

[9] R. E. Brown, J. Pan, X. Feng, and K. Koutlev, 2001, "Siting distributed generation to defer T\&D expansion", Proceedings IEEE/PES Transmission and Distribution Conference and Exposition, vol. 2, 622-627.

[10] G. W. Ault and J. R. McDonald, 2000, "Planning for distributed generation within distribution networks in restructured electricity markets", IEEE Power Engineering Review, vol. 20, no. 2, 52-54.

[11] A. J. Conejo, F. D. Galiana, and I. Kockar, 2001, "Z-bus loss allocation", IEEE Trans. o Power Systems, vol. 16, no. $1,105-110$

[12] W. H. Kersting, 1991, "Radial distribution test feeders", IEEE Trans. on Power Systems, vol. 6, no. 3, 975-985.

[13] R. M. Ciric, A. Padilha, and L. F. Ochoa, 2003, "Power flow in four-wire distribution networks-general approach", IEEE Trans. on Power Systems, vol. 18, no. 4, 12831290 . 